\title{
Should ECG be required in young athletes?
}

Athletes' hearts are adapted to chronic exercise training that may result in physiological increases in left-ventricular volume, left-ventricular hypertrophy, increased left-atrial volume, and right-ventricular structural changes, with preserved systolic and diastolic ventricular function and exercise ability. ${ }^{1,2}$ The extent and variability of cardiac morphological changes in trained athletes varies with the type of exercise training, age, gender, environment, and body size. Genotype and different polymorphisms of the angiotensin-converting enzyme or angiotensinogen genes can result in different phenotypic expression in athletes' hearts.

Results of resting 12-lead electrocardiogram (ECG) reflect the autonomic and structural changes resulting from training. ${ }^{3,4}$ Increased vagal tone usually results in sinus bradycardia, and can be associated with certain types of benign cardiac arrhythmias, ventricular hypertrophy, and repolarisation changes, such as ST segment elevation of the early repolarisation type and biphasic or inverted $T$ waves, which occur in $2-4 \%$ of trained athletes aged 18-35 years. ${ }^{3}$ The commonness of ECG abnormalities in apparently healthy athletes with above average exercise performance contrasts sharply with the rareness of major cardiac pathology in these individuals and extremely rare incidents of sudden cardiac death. ${ }^{5}$

The requirement for an ECG (and in some countries, an echocardiogram and exercise test) to detect underlying cardiac pathology in young, apparently healthy athletes is controversial. In many European countries, not only is a careful history and physical examination needed, but also a 12-lead resting ECG is required before allowing competition, a position endorsed by the Lausanne recommendations for the International Olympic Committee (IOC).${ }^{6,7}$ By contrast, the American Heart Association does not require or recommend routine 12-lead ECG as part of the precompetition assessment, unless the athlete fails a prescreening questionnaire and physical examination. ${ }^{8}$ The position of the American Heart Association has recently been debated. ${ }^{9,10}$

The Summer Olympics will be held in Beijing, China, in August 2008. The decision to do an ECG as a precompetition requirement would have been considered by National Olympic Committees and International Federations well before the Beijing Olympics. However, how much prognostic accuracy a resting ECG adds to the detection of rare cardiac pathology and even more rare cardiac events that result in sudden cardiac death in apparently healthy athletes is unclear.

In a recent series, Antonio Pelliccia and colleagues ${ }^{11}$ described the clinical course of 123 (1\%) of 12550 trained athletes who had inverted $\mathrm{T}$ waves equal to or greater than $2 \mathrm{~mm}$ in at least three leads, predominantly V2-V6, without apparent cardiac disease; 81 subsequently had serial clinical, ECG, and echocardiographic studies. ${ }^{11}$ The athletes were followed up for a mean of 9 years (range 1-27 years) and were compared with 229 athletes matched for age and sex with normal ECG followed up for a similar time. Of the 81 athletes, $5(6 \%)$ ultimately developed a cardiomyopathy, including one who died suddenly at age 24 years from arrhythmogenic right-ventricular

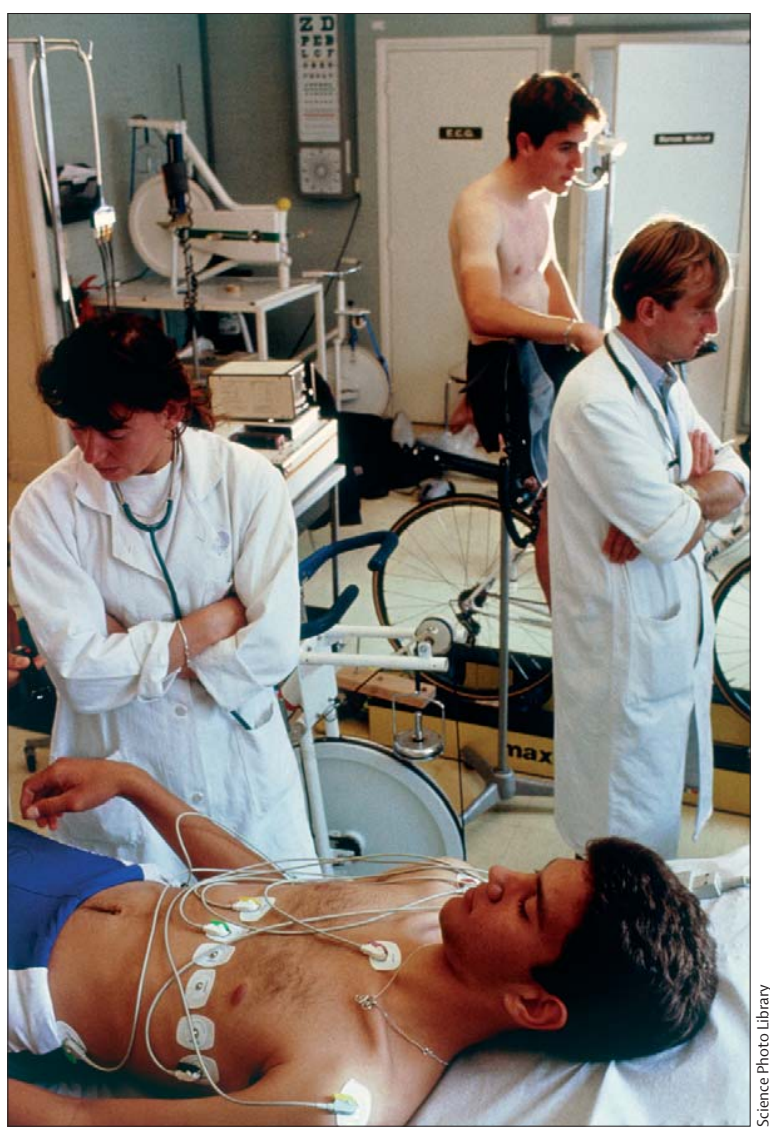


dysplasia, versus none in the control group. The prevalence of congenital defects in the 12550 athletes was $0.03 \%$ and there were sudden cardiac arrests in two patients $(0.01 \%)$, one of whom was resuscitated and the other of whom died.

Basavarajaiah and co-workers assessed the prevalence of hypertrophic cardiomyopathy in 3500 elite British athletes by use of clinical history, physical examination, screening ECG, and echocardiogram. ${ }^{12} 1 \%$ had deep T-wave inversion and $1.5 \%$ had echocardiographic evidence of left-ventricular hypertrophy. Physiological adaptation to training explained the findings in 50; only three $(0.08 \%)$ had left-ventricular hypertrophy with non-dilated left ventricles and associated T-wave inversion, each of whom had normal cardiac MRI, normal right-ventricular and left-ventricular structure and function, and normal 48-h ambulatory ECG monitoring. One athlete who agreed to stop training had normal findings after 12 weeks.

Routine non-invasive testing with an echocardiogram is a cost-inefficient way to prospectively screen highly trained athletes, particularly those able to compete at Olympic level. The role of the resting ECG, a less expensive modality, as a prescreening requirement for different types of competitive sports in all younger athletes remains controversial, 9,10 and the use of mandatory self-funded non-invasive test screening varies among different sports associations. Additional research into methods to cost-effectively screen people involved in high-level exercise activities would be valuable.

\section{${ }^{*}$ Bernard R Chaitman, Martin Fromer}

Department of Medicine, Division of Cardiology, Saint Louis University School of Medicine, St Louis, MO 63117, USA (BRC) and Department of Medicine, Cardiology Service, Arrhythmia Unit, University Hospital CHUV, Lausanne, Switzerland (MF) chaitman@slu.edu

We declare that we have no conflict of interest.

1 Maron BJ, Pelliccia A. The heart of trained athletes: cardiac remodeling and the risks of sports, including sudden death. Circulation 2006; 114: 1633-44.

2 Atchley AE Jr, Douglas PS. Left ventricular hypertrophy in athletes: morphologic features and clinical correlates. Cardiol Clin 2007; 25: 371-82.

3 Pelliccia A, Maron BJ, Culasso F, et al. Clinical significance of abnormal electrocardiographic patterns in trained athletes. Circulation 2000 102: $278-84$

4 Ferst JA, Chaitman BR. The electrocardiogram and the athlete. Sports Med 1984; 1: 390-403.

5 Pelliccia A. The preparticipation cardiovascular screening of competitive athletes: is it time to change the customary clinical practice? Eur Heart 2007; 28: 2703-05.

6 Corrado D, Pelliccia A, Bjornstad HH, et al. Cardiovascular preparticipation screening of young competitive athletes for prevention of sudden death: proposal for a common European protocol: consensus statement of the Study Group of Sport Cardiology of the Working Group of Cardiac Rehabilitation and Exercise Physiology and the Working Group of Myocardial and Pericardial Diseases of the European Society of Cardiology. Eur Heart J 2005; 26: 516-24.

7 IOC Medical Commission, International Olympic Committee. Sudden cardiovascular death in sport: Lausanne recommendations. Dec 10, 2004. http://multimedia.olympic.org/pdf/en_report_886.pdf (accessed Feb 21, 2008)

8 Maron BJ, Thompson PD, Ackerman MJ, et al. Recommendations and considerations related to preparticipation screening for cardiovascular abnormalities in competitive athletes: 2007 update-a scientific statement from the American Heart Association Council on Nutrition, Physical Activity and Metabolism. Circulation 2007; 115: 1643-55.

9 Chaitman BR. An electrocardiogram should not be included in routine preparticipation screening of young athletes. Circulation 2007; 116: 2610-15.

10 Myerburg RJ, Vetter VL. Electrocardiograms should be included in preparticipation screening of athletes. Circulation 2007; 116: 2616-26.

11 Pelliccia A, Di Paolo FM, Quattrini FM, et al. Outcomes in athletes with marked ECG repolarization abnormalities. N EnglJ Med 2008; 358: 152-61.

12 Basavarajaiah S, Wilson M, Whyte G, Shaw A, McKenna W, Sharma S. Prevalence of hypertrophic cardiomyopathy in highly trained athletes. J Am Coll Cardiol 2008; 51: 1033-39.

\section{Expression of concern-autologous myoblasts and fibroblasts for treatment of stress urinary incontinence: a randomised controlled trial}

On April 22, it was brought to our attention that the published trial registration number provided by the authors of this study is incorrect. ${ }^{1}$ We have also been made aware of concerns about the ethical approval and conduct of this study, which are currently subject to investigations in Austria. Pending the outcome of these investigations, we issue an expression of concern about the article by Dr Hannes Strasser and colleagues. ${ }^{1}$

\section{The Editors of The Lancet}

1 Strasser $\mathrm{H}$, Marksteiner R, Margreiter E, et al. Autologous myoblast and fibroblasts versus collagen for treatment of stress urinary incontinence in women: a randomised controlled trial. Lancet 2007; 369: $2179-86$ 